\title{
Massive Bitemporal Hemorrhages in Antineutrophil Cytoplasmic Autoantibodies Vasculitis
}

\author{
David Bergeron, Annie Dionne, Raphaël Marchand, Robert Jr Laforce
}

Keywords: Vasculitis, Cerebral hemorrhage, Neuroimaging

doi:10.1017/cjn.2016.401

Can J Neurol Sci. 2017; 44: 194-195

ANCA-associated small vessel vasculitides are systemic diseases characterized by inflammation and necrosis of small-sized blood vessels. ${ }^{1}$ They are characterized by the presence of antineutrophil cytoplasmic antibodies (ANCAs), which primarily target the kidney, lung, skin and peripheral nervous system. ${ }^{2}$ In rare cases, they can affect the central nervous system (CNS) and cause cerebrovascular events. ${ }^{3}$ We present a patient with ANCA vasculitis who developed fatal massive bitemporal hemorrhages during the course of immunosuppressive therapy.

A 71-year-old man was referred to our tertiary neurological center due to weight loss (10 kg in two months), fatigue, weakness and persistent fever of unknown origin. The patient did not report previous sinusitis, upper airway infection, arthritis or hematuria. Neurological examination only revealed a right foot drop. Laboratory studies showed inflammatory anemia and elevated inflammatory markers. Screening for infectious diseases was negative. Spine MRI indicated a herniated L5-S1 disc. Brain MRI was normal except for a millimetric left occipital ischemic infarct. Whole-body PET scan was conducted to eliminate a neoplastic etiology, but was also negative. Five days after his arrival, the patient developed new weaknesses and paresthesia in the left leg and right hand. Laboratory studies revealed acute renal failure (creatinine $133 \mu \mathrm{mol} / \mathrm{L}$, hematuria, proteinuria) and elevated c-ANCA counts (1:320). A second MRI of the brain conducted 10 days after the first showed five new millimetric ischemic foci in the right temporal and left cerebellar regions. In concert with rheumatology, a diagnosis of ANCA vasculitis was made, an immunosuppressive treatment was initiated (high-dose intravenous glucocorticoids), and the patient's neurological symptoms were stabilized. However, creatinine continued to rise (from 133 to $177 \mu \mathrm{mol} / \mathrm{L}$ in 7 days). A renal biopsy was scheduled to confirm a diagnosis of glomerulonephritis. After five days of induction treatment, tapering oral doses of prednisone were administered. On the first day of prednisone tapering, the patient was found unresponsive in his bed (eyes opened, but unresponsive to simple commands). Head CT showed massive bilateral temporal hemorrhages (see Figure 1). After discussion with the family, the patient was transferred back to his hometown for end-of-life care. Renal biopsy and autopsy could not be performed. Nevertheless, a recent meta-analysis has evaluated the specificity of elevated c-ANCA levels in the serum at $96.3 \%$ (CI [94.1-98.5]), ${ }^{4}$ which, combined with the high pretest probability of vasculitis (mononeuritis multiplex, acute renal failure, inflammatory anemia,

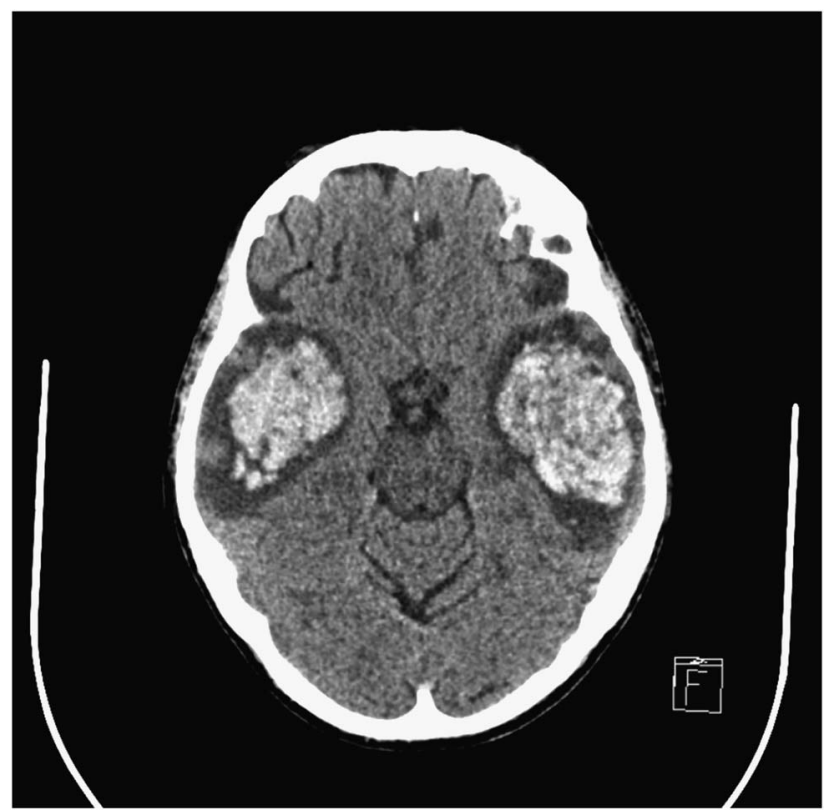

Figure 1: Axial view of a head CT showing massive bitemporal haemorrhages with significant mass effect.

multifocal brain infarcts), made a diagnosis of ANCA vasculitis highly probable even without a gold-standard pathological diagnosis.

While it is known that ANCA vasculitis may in some instances involve the $\mathrm{CNS},{ }^{3,4}$ only a few case reports describe the appearance of cerebral hemorrhages, ${ }^{5-10}$ and even fewer a rapid progression toward massive hemorrhages and death. ${ }^{5-7}$ Altogether, our case supports the possibility of cerebral

From the Clinique Interdisciplinaire de Mémoire (CIME), Centre Hospitalier Universitaire de Québec, Québec City, Québec, Canada (DB, AD, RM, RJL); Département des Sciences Neurologiques, Université Laval, Québec City, Québec, Canada (DB, AD, RM, RJL).

Received September 29, 2015. Final Revisions Submitted March 3, 2016. Date of ACCEPTANCE APRIL 23, 2016.

Correspondence to: David Bergeron, Clinique Interdisciplinaire de Mémoire (CIME), CHU de Québec, 1401, 18ième rue, Québec, Canada, G1J 1Z4. Website: www. cliniquedememoire.ca. E-mail: david.bergeron.5@ulaval.ca. 
vasculitis, though rare in ANCA-positive patients, causing so massive a cerebral hemorrhage.

\section{ACKNOWLEDGMENTS}

We thank the patient's family for their collaboration. We also thank Audrey Paradis for manuscript formatting. Oral and written consent was obtained from the patient's family.

\section{Statement of Authorship}

David Bergeron drafted the initial version of the manuscript. Dr. Annie Dionne was the treating physician. All authors contributed equally to the writing process and approved the final version of the manuscript.

\section{Disclosures}

David Bergeron, Raphaël Marchand and Robert Jr Laforce do not have anything to disclose.

Annie Dionne has the following disclosures: Teva: conducting clinical trials, research grant for the conduct of clinical trials; Novartis: conducting clinical trials, research grant for the conduct of clinical trials.

\section{REFERENCES}

1. Jennette JC, Falk RJ. Small-vessel vasculitis. N Engl J Med. 1997;337(21):1512-23.

2. Falk RJ, Jennette JC. Anti-neutrophil cytoplasmic autoantibodies with specificity for myeloperoxidase in patients with systemic vasculitis and idiopathic necrotizing and crescentic glomerulonephritis. N Engl J Med. 1988;318(25):1651-7.

3. Nishino H, Rubino FA, DeRemee RA, Swanson JW, Parisi JE. Neurological involvement in Wegener's granulomatosis: an analysis of 324 consecutive patients at the Mayo Clinic. Ann Neurol. 1993;33(1):4-9.

4. Choi HK, Liu S, Merkel PA, Colditz GA, Niles JL. Diagnostic performance of antineutrophil cytoplasmic antibody tests for idiopathic vasculitides: metaanalysis with a focus on antimyeloperoxidase antibodies. J Rheumatol. 2001;28(7):1584-90.

5. Akkara Veetil BM, Schimmer BM. A case of limited systemic sclerosis with p-ANCA, complicated by multiple cerebral hemorrhages. Rheumatol Int. 2009;29(3):325-9; Epub ahead of print Aug 22, 2008.

6. Ito Y, Suzuki K, Yamazaki T, Yoshizawa T, Ohkoshi N, Matsumura A. ANCA-associated vasculitis (AAV) causing bilateral cerebral infarction and subsequent intracerebral hemorrhage without renal and respiratory dysfunction. J Neurol Sci. 2006;240(1-2):99-101; Epub ahead of print Nov 7, 2005.

7. Sasaki A, Hirato J, Nakazato Y, Tanaka T, Takeuchi H. An autopsy case of P-ANCA-positive microscopic polyangiitis with multiple cerebral hemorrhagic infarction [in Japanese]. No To Shinkei. 1998;50(1):56-60.

8. Gandhi S, Fazeli P. Intracranial hemorrhage due to ANCAassociated isolated cerebral vasculitis. Minn Med. 2015;98(4):43.

9. Yamashiro K, Mori A, Shimada Y, Furuya T, Noda K, Urabe T, Hattori N, et al. Gradient echo T2*-weighted magnetic resonance imaging revealing cerebral microbleeds in a patient with microscopic polyangiitis complicated by cerebrovascular disease. J Stroke Cerebrovasc Dis. 2012;21(8):904 e7-9; Epub ahead of print Jun 2, 2011.

10. Han S, Rehman HU, Jayaratne PS, Carty JE. Microscopic polyangiitis complicated by cerebral haemorrhage. Rheumatol Int. 2006;26(11):1057-60; Epub ahead of print Jun 2. 